\title{
Experimental Validation of a SAR-Based RFID Localization Technique Exploiting an Automated Handling System
}

\author{
Alice Buffi, Marcos Rodriguez Pino, and Paolo Nepa
}

\begin{abstract}
The Synthetic Aperture Radar (SAR) approach has attracted a considerable interest in the context of phase-based techniques for the localization of UHF-RFID passive tags. In this letter, the results of an extensive experimental activity are presented, when the reader antenna is moved in front of a set of static passive UHF-RFID tags by means of a planar handling system. Measured performance is evaluated with respect to several system parameters: tag number and typology, tag reciprocal distance and orientation, trajectory and speed of the moving reader antenna. It is shown that the SAR processing can guarantee an accurate two-dimensional localization of multiple tags, with a localization error comparable to the size of typical commercial tags, by using a single reader antenna and without the need for any reference tag.
\end{abstract}

Index terms-Phase-based tag localization, RFID tag localization, synthetic aperture radar (SAR)-based tag localization, tagged item localization.

\section{INTRODUCTION}

$\mathrm{T}_{\mathrm{le}}^{\mathrm{h}}, \mathrm{ars}$ The passive UHF-RFID technology represents a low-cost and easily deployable solution to combine real-time inventory with localization and tracking of tagged items, in several scenarios such as warehouses, libraries, and hospitals. Thus, a large number of tagged items can be concurrently managed and the service processes can be faster and more efficient.

Satisfactory localization performance can be obtained with phase-based methods [1], which are more robust than amplitudebased approaches with respect to the multipath propagation phenomena that are typical of any crowded indoor scenario. In this context, multiple reader antennas can be employed to increase the amount of data available for the localization processing [2], [3]. Alternatively, a synthetic aperture radar (SAR)-based approach can be adopted [4], [9], which exploits the relative motion between the reader antenna and the tag. In [4] and [6], the SAR-based approach has been applied to localize parcels or bags moving along a conveyor belt with respect to the reader antenna of a fixed RFID portal. In [7] and [9], it has been applied to locate fixed tagged items by employing a reader antenna moved by a handling system. Indeed, in those scenarios where an automated handling system for item management is already available, the reader installation can be easily performed, as for example in warehouses equipped with overhead travelling cranes, monorails or hoists. The SAR approach combined with an automated handling system may represent a more appealing solution with respect to the installation of multiple reader antennas, especially when large warehouses are considered. It is worth noting that the above mentioned scenario is dual with respect to that one where a robot equipped with a reader antenna is localized with respect to a set of fixed reference tags [10].

In this letter, an experimental setup equipped with a planar antenna handling system is used to measure the performance of a SAR-based RFID localization technique. The planar scanning system of a compact anechoic chamber for near-field antenna characterization is exploited to show the two-dimensional (2-D) localization capabilities. The advantages of a $2-\mathrm{D}$ scanning versus a $1-\mathrm{D}$ scanning are shown. The localization performance is evaluated in a multiple-tag scenario, for different tag number, typology, reciprocal distance and orientation. In addition, different planar scanning paths and reader antenna speeds are used in the data acquisition procedure. The phase samples have been processed through a basic SAR algorithm, and implementation details can be found both in [5] and [9].

\section{MEASUREMENT SETUP}

The measurement campaign has been carried out in the near-field antenna test range at the University of Oviedo, Gijón, Spain. To move the reader antenna, the measurement setup uses the planar scanning system of a compact $1.5 \mathrm{~m} \times 1.5 \mathrm{~m} \times 0.4 \mathrm{~m}$ anechoic chamber (see Fig. 1).

The handling system carries a circularly polarized reader antenna (WANTENNAX005 by C.A.E.N. RFID) that is connected to the Impinj Speedway R420 Revolution UHF-RFID reader. The reader antenna is moved on a plane parallel to the $x y$ plane (see the reference system in Fig. 1), at a height from the chamber floor, $h_{\text {ant }}$, that can vary between 0.75 and $1.15 \mathrm{~m}$. The scan trajectory is programmable and the maximum speed is $v=15 \mathrm{~cm} / \mathrm{s}$. The operating frequency of $f_{0}=865.7 \mathrm{MHz}$ (ETSI channel 4) has been set. The adopted planar scanning system allows for the knowledge of the reader antenna instantaneous position at the 
time of each tag reading (as given by the reader timestamp), which is a parameter required for the application of the SAR processing.

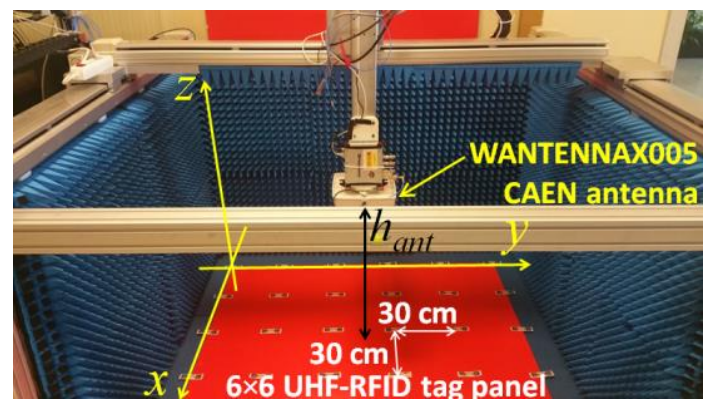

(a)

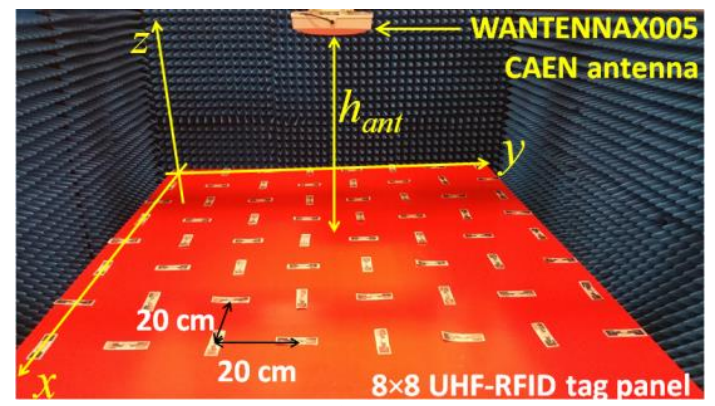

(b)

Fig. 1 - Near-field antenna test range with a tag panel on the floor of a compact anechoic chamber: (a) $6 \times 6$ tags at a relative distance of $d_{\text {tag }}=30 \mathrm{~cm}$ and horizontally oriented (parallel to $y$-axis). (b) $8 \times 8$ tags at a relative distance of $d_{t a g}=20 \mathrm{~cm}$, which are alternatively horizontally and vertically oriented. The reader antenna is carried by the planar scanning system at a distance from the tag panel that can vary between 0.75 and $1.15 \mathrm{~m}$.

A panel with $N_{\text {tag }} \times N_{\text {tag }}$ tags has been arranged on the floor of the anechoic chamber (see Fig. 1). The tag $(m, n)$ is located at $p_{\text {tag }}=\left[x_{\text {tag }}, y_{\text {tag }}\right]=\left[(m-1) d_{\text {tag }},(n-1) d_{\text {tag }}\right]$, where $n, m=\left\{1, \ldots, N_{\text {tag }}\right\}$, and $d_{\text {tag }}$ is the tag separation distance along both principal axes. Two different tag distributions have been considered: 1) 6×6 tags (Smartrac-Dogbone MZ4 Paper Face tags with -17.4 dBm chip sensitivity) are positioned at a relative distance of $d_{t a g}=30 \mathrm{~cm}$, and they are all parallel oriented to the $y$-axis [see Fig. 1(a)]; 2) $8 \times 8$ tags (36 Smartrac-Dogbone MZ4 Paper Face tags and 28 Smartrac-Dogbone MZ-R6 wet Inlay tags with -20 dBm chip sensitivity) are positioned at a relative distance of $d_{t a g}=20 \mathrm{~cm}$, and they are alternatively oriented along the $x$-axis and the $y$-axis [see Fig. 1(b)].

An L-scan of the reader antenna has been firstly considered (see Fig. 2). The antenna starts at the point $[0.05,1.45] \mathrm{m}$, runs parallel to the $y$-direction and stops for around $1 \mathrm{~s}$ at the position [0.05, 0.05] $\mathrm{m}$ (trajectory corner); then, it runs parallel to the $x$ direction and stops at $[1.45,1.45] \mathrm{m}$. As for the configuration with $6 \times 6$ tags shown in Fig. 1a, the relative phase history measured at the reader side is illustrated in Fig. 3, for three different tags. The observation interval is the same for the horizontal and vertical parts of the L-scan: $T_{x} \sim T_{y} \sim 9.6 \mathrm{~s}$.

The reader antenna stops at the trajectory corner cause a set of constant phase samples that are not considered in the SAR processing (this is the reason for the missed samples around $t=10 \mathrm{~s}$, in Fig. 3). Due to the relatively low speed of the reader antenna, a large number of useful readings can be collected for each tag: 166 for tag $(1,1), 204$ for tag $(2,5)$ and 208 for tag $(6$, 6). It is worth noting that the tag phase history usually exhibits a nonsymmetric profile, except for the tags that lie along the diagonal of the first quadrant (as for example tag $(6,6)$ in Fig. 3). By the knowledge of the reader antenna trajectory and the actual tag position, it is possible to calculate the expected theoretical phase history (curves without markers in Fig. 3). The quite good agreement between such theoretical phase histories and the measured phase data confirms that the phase samples of the tagbackscattered signal can be fruitfully employed to estimate the tag position in a $2 \mathrm{D}$ plane. Specifically, by using the notation as in [5], the tag position can be estimated as:

$$
p_{\text {tag }}=\underset{p_{\text {tag }}{ }^{\prime}}{\arg \max } \frac{\left|\boldsymbol{a}^{\boldsymbol{H}}\left(p_{\text {tag }}{ }^{\prime}\right) \boldsymbol{y}_{p}\right|}{\left\|\boldsymbol{a}^{H}\left(p_{\text {tag }}{ }^{\prime}\right)\right\|^{2} \cdot\left\|\boldsymbol{y}_{p}\right\|^{2}},
$$

Where $p_{t a g}=\left[x_{t a g}, y_{t a g}\right]$ is the vector of the hypothetical tag $2-\mathrm{D}$ coordinates, $\boldsymbol{a}^{\boldsymbol{H}}=\left(p_{t a g}{ }^{\prime}\right)$ is the sampled nominal phase history and $\boldsymbol{y}_{\boldsymbol{p}}$ is the vector of the measured phase samples.

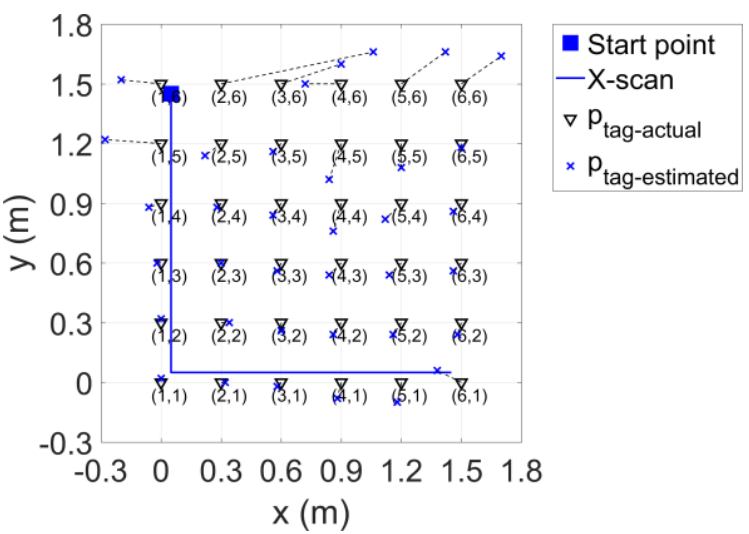

Fig. 2 - L-scan trajectory run by the reader antenna during the phase sample acquisition, when the $6 \times 6$ tag configuration in Fig. 1(a) is considered. The figure also presents the tag actual positions (triangle marker) and the tag estimated positions (cross marker) after applying the SAR processing to a set of phase data acquired during the L-scan (see Fig. 3) for the following system parameters: $f_{0}=865.7 \mathrm{MHz}, v=15 \mathrm{~cm} / \mathrm{s}, h_{a n t}=1.15 \mathrm{~m}$. 


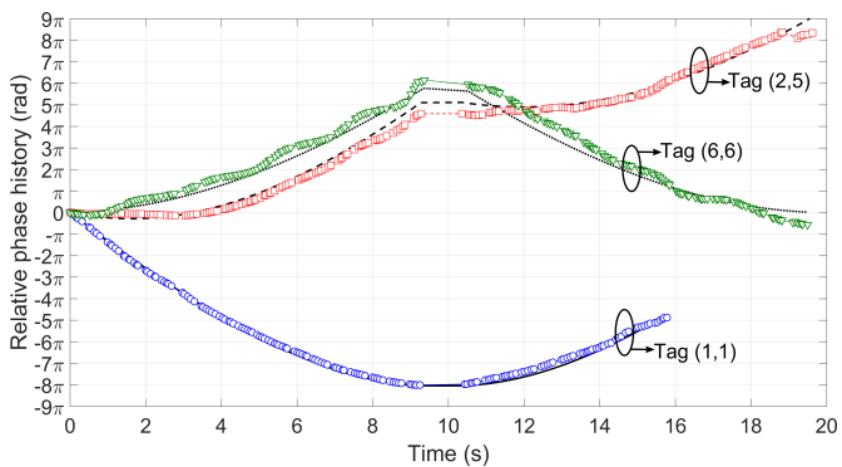

Fig. 3 - Measured phase samples during the L-scan, for tag $(1,1)$ (circle blue marker), tag $(2,5)$ (square red marker) and tag $(6,6)$ (triangle green marker), which are placed at $\left[x_{\text {tag }}, y_{\text {tag }}\right]=[0,0] \mathrm{m},\left[x_{\text {tag }}, y_{\text {tag }}\right]=[0.6,1.2] \mathrm{m}$ and $\left[x_{\text {tag }}, y_{\text {tag }},\right]=[1.5,1.5] \mathrm{m}$, respectively. For each tag, the theoretical phase history has been also added (curves without markers). The following system parameters have been considered: $f_{0}=865.7 \mathrm{MHz}, v=15 \mathrm{~cm} / \mathrm{s}, h_{\text {ant }}=1.15 \mathrm{~m}$.

\section{LOCALIZATION PERFORMANCE IN MULTIPLE-TAGS SCENARIO}

Let us consider the two sections of the curve relative to the phase history of tag $(1,1)$ in Fig. 3: the first one, with decreasing phase values, corresponds to the antenna running parallel to the $y$-axis and approaching the tag; the second one, with increasing phase values, corresponds to the scan parallel to the $x$-axis. The 2-D SAR processing (1) has been applied separately to either each rectilinear scan (parallel to $y$-axis or $x$-axis) or the whole L-scan. Fig. 4 shows the 2-D normalized matching function in (1) versus the hypothetical tag coordinates in the $x y$ plane $\left[x_{t a g}, y_{t a g}{ }^{\prime}\right]$, for the three different cases. The peak position determines the estimated tag position in the 2D space: $\left[x_{t a g}, y_{t a g}\right]$. The SAR processing along a rectilinear direction [see Fig. 4(a) and Fig. 4(b)] determines a larger uncertainty in the estimation of the coordinate orthogonal to the scan direction, as apparent from the wide main lobe. In particular, when considering only the rectilinear scan along the $y$-axis [see Fig. 4a)], the location errors are equal to $\varepsilon_{x}=\left|x_{\text {tag }}-x_{\text {tag }}\right|=56 \mathrm{~cm}$ and $\varepsilon_{y}=\left|y_{\text {tag }}-y_{\text {tag }}\right|=10 \mathrm{~cm}$.

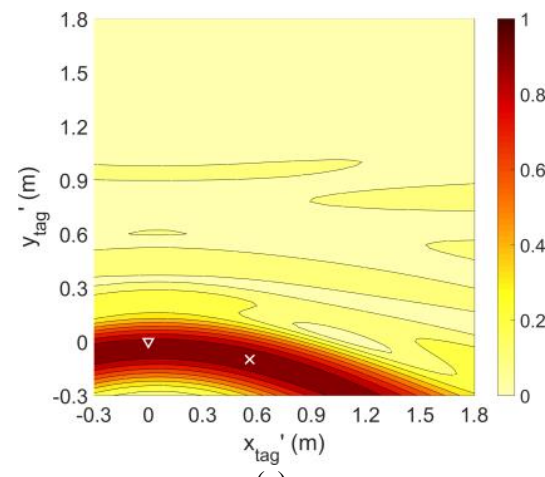

(a)

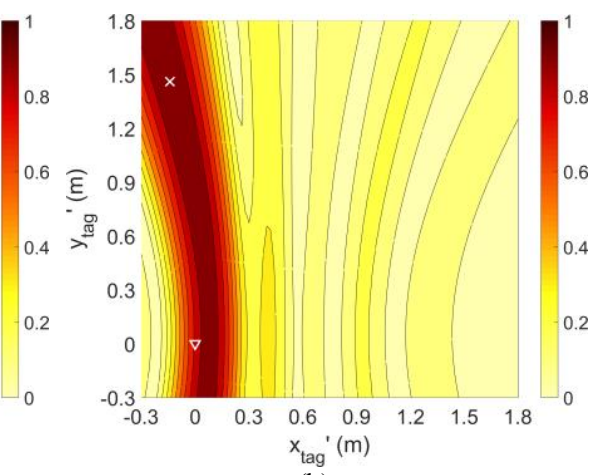

(b)

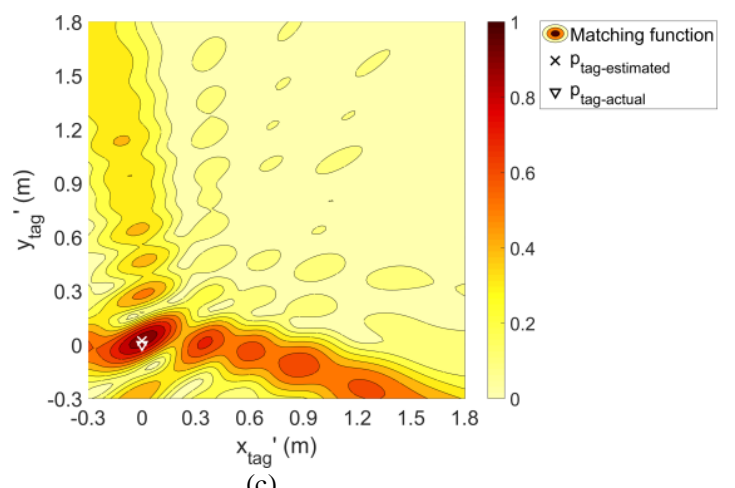

(c)

Fig. 4. 2-D matching function of tag $(1,1)$ located at $\left[x_{t a g}, y_{t a g}\right]=[0,0] \mathrm{m}$ when considering: (a) a rectilinear scan along the $y$-axis, (b) a rectilinear scan along the $x$-axis, and (c) the whole L-scan. Other system parameters are as in Fig. 3.

As the rectilinear scan along the $x$-axis [see Fig. 4(b)], the location errors are equal to $\varepsilon_{x}=14 \mathrm{~cm}$ and $\varepsilon_{y}=146 \mathrm{~cm}$. On the other hand, by processing the phase samples collected during the whole L-scan, a very distinctive peak appears on the $x y$ plane and both coordinates are estimated with a lower error: $\varepsilon_{x}=0.5 \mathrm{~cm}$ and $\varepsilon_{y}=2 \mathrm{~cm}$ [see Fig. 4(c)]. Thus, the SAR processing of the wholepath phase history improves the localization performance, as expected from a 2-D scanning [8], [9].

Localization performance for all the $6 \times 6$ tags is presented in Fig. 2, when all phase samples acquired during the entire L-scan are processed. The mean values of the localization errors are $\mu_{x}=8.7 \mathrm{~cm}$ and $\mu_{y}=5.8 \mathrm{~cm}$, and they can be considered as satisfactory in many applications as they are comparable with the tag size that is around $10 \mathrm{~cm}$.

The achievable spatial resolution (matching function monolateral width) and accuracy (peak position with respect to the actual tag position) are strictly dependent on the path run by the antenna, namely on the synthetic aperture size $D$ [5]. For a rectilinear synthetic array, the resolution can be approximated as $R=r_{M} \lambda / 2 D$, where $r_{M}$ is the minimum distance between the tag and the antenna trajectory [5]. Thus, greater the synthetic aperture length, lower the spatial resolution. For an assigned 2-D scan of the reader antenna, the effective synthetic aperture size varies from tag to tag, as it actually depends on the tag location with respect to the antenna path. For example, for the above considered L-scan and the $6 \times 6$ tag configuration, the maximum value of the effective synthetic aperture size is $D_{x}=D_{y}=1.4 \mathrm{~m}$ (namely the size of the L-scan along the two axes), while its mean value is equal to $\mu_{D x}=1.26 \mathrm{~m}$ with a minimum value of $\min \left\{D_{x}\right\}=0.58 \mathrm{~m}$, and $\mu_{D_{y}}=1.36 \mathrm{~m}$ with a minimum value of $\min \left\{D_{y}\right\}=1.16 \mathrm{~m}$, for the $x$ coordinate and $y$-coordinate, respectively. The difference between the above values for the two coordinates justifies the lower 
location error of the tag $y$-coordinate. The worst cases are associated to tags that are at a larger distance from the antenna trajectory for which the system resolution degrades since it is directly proportional to the minimum distance between the reader antenna trajectory and the tag [5].

Measurements have been repeated by considering different reader antenna heights with respect to the tag panel, within the range $h_{a n t}=[75,115] \mathrm{cm}$. Results are illustrated in Fig. 5 for both tag panel configurations in Fig. 1. For the $6 \times 6$ tag arrangement, the location errors are less than $13 \mathrm{~cm}$, while for the $8 \times 8$ tag configuration they are less than $16 \mathrm{~cm}$, for both coordinates.

Furthermore, the effect of the reader antenna speed has been analyzed. When increasing the speed from $v=5 \mathrm{~cm} / \mathrm{s}$ to $\nu=15 \mathrm{~cm} / \mathrm{s}$, the mean value of the response interval (averaged on all tags) decreases from 57 to $20 \mathrm{~s}$ and the spatial sampling of the measured phase history roughly changes from $\Delta s=0.5 \mathrm{~cm}$ to $\Delta s=1.5 \mathrm{~cm}$. Since the antenna path length does not change, we expect that even the synthetic array length for each tag remains almost the same, so guaranteeing similar localization performance, as shown in Fig. 6. Generally speaking, the SAR processing can be applied with a higher speed of the reader antenna, provided that the spatial sampling of the available tag responses is less than a quarter-wavelength [5], [9]. Fig. 6 also shows the mean value of the localization error when different antenna trajectories are considered: L-scan, X-scan and Squarescan (the path relevant to the last two scans are shown in Fig. 7). The localization performance is almost independent on the antenna path (location error less than $14 \mathrm{~cm}$ ), demonstrating that the SAR-based technique can be successfully implemented for almost any 2-D reader antenna trajectory. It is worth mentioning that a precise synchronization of the reader and the personal computer controlling the automated handling system is an important requirement, as the SAR-based approach needs for an accurate knowledge of the reader antenna position at the time of the tag reading (reader timestamp).

To evaluate the effect of relatively strong multipath phenomena, a metal sheet has been placed under the tag panel at two different distances: 22 and $30 \mathrm{~cm}$. The localization error is illustrated in Fig. 8 for a speed of $v=10 \mathrm{~cm} / \mathrm{s}$, for different trajectories of the reader antenna. The metal sheet mainly affects the localization performance at the smaller distance of $22 \mathrm{~cm}$. However, the localization error is lower than $25 \mathrm{~cm}$, thus confirming the expected robustness of the SAR-based technique to multipath phenomena.

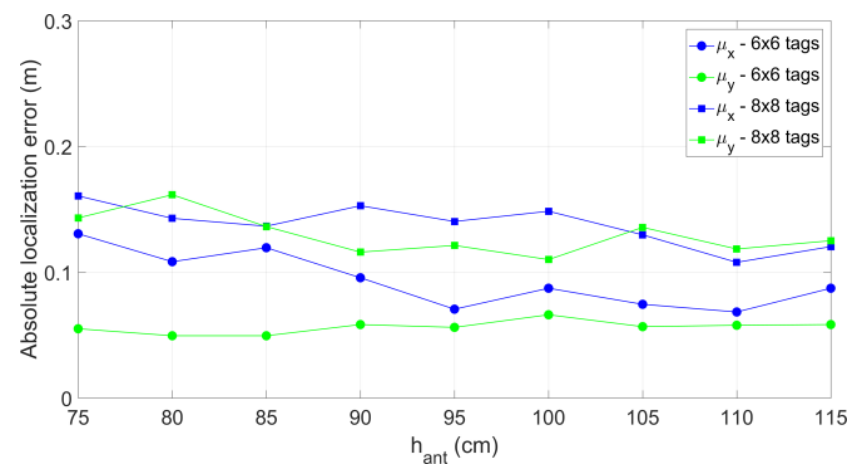

Fig. 5. Localization error versus the reader antenna height from the tag panel $\left(h_{a n t}\right)$, for the reader antenna performing an L-scan: $6 \times 6$ tag $($ circle marker) and $8 \times 8$ tag (square marker) configurations. Other system parameters are as in Fig. 3.

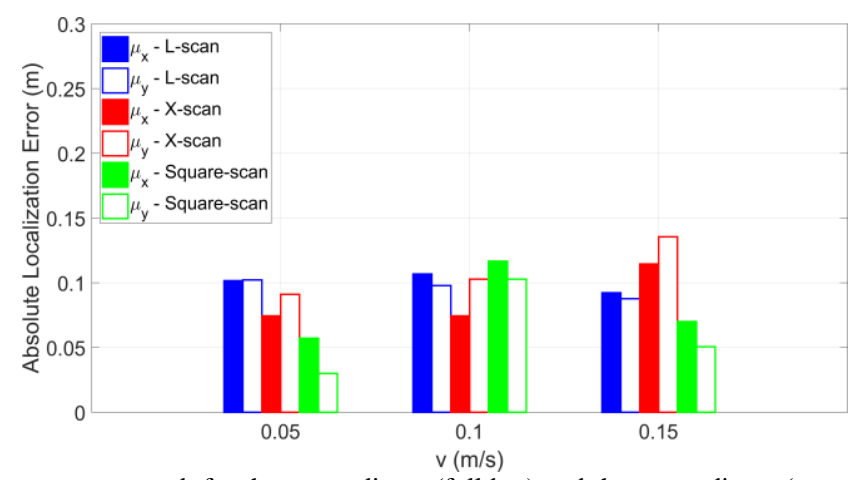

Fig. 6. Localization error versus the reader antenna speed, for the $x$-coordinate (full bar) and the $y$-coordinate (empty bar), for different scanning paths: L-scan (blue bar), X-scan (red bar) and Square-scan (green bar). The $6 \times 6$ tag configuration has been employed with an antenna height of $h_{\text {ani }}=0.85 \mathrm{~m}\left(f_{0}=865.7 \mathrm{MHz}\right)$. $\mathrm{X}$-scan and Square-scan paths are shown in Fig. 8. 

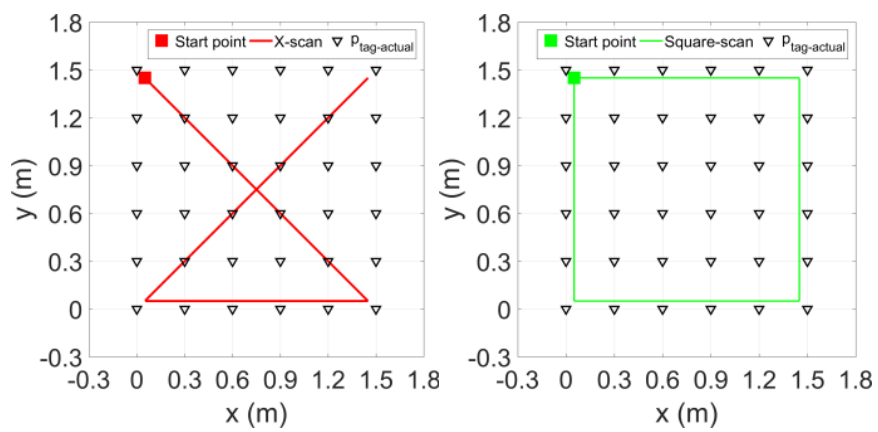

Fig. 7. X-scan (left) and Square-scan (right) of the reader antenna.

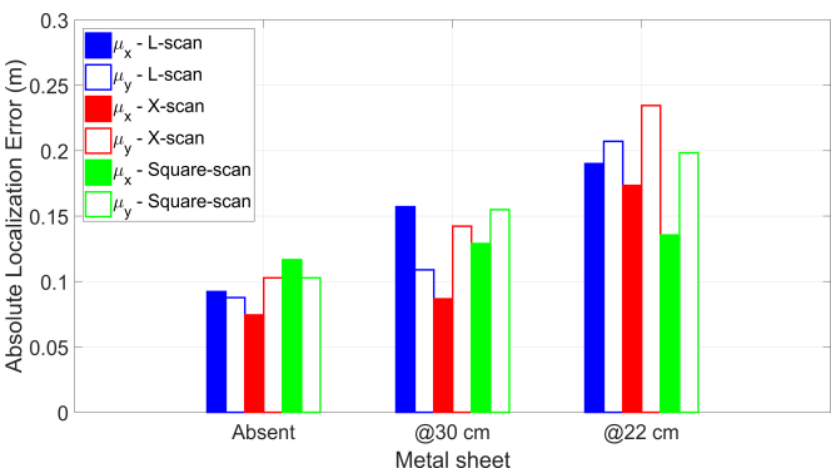

Fig. 8. Localization error with respect to the presence of a metal sheet under the tag panel, for different antenna scanning paths: L-scan (blue bar), X-scan (red bar) and Square-scan (green bar). The $6 \times 6$ tag configuration has been employed, with an antenna speed of $v=10 \mathrm{~cm} / \mathrm{s}$ and height of $h_{\text {ant }}=0.85 \mathrm{~m}\left(f_{0}=865.7 \mathrm{MHz}\right)$.

\section{CONCLUSION}

In the context of the phase-based techniques for the localization of UHF-RFID passive tags, the measurement results presented in this paper have confirmed that a SAR-based approach can guarantee localization precision comparable to the size of typical commercial tags. Measured performance has been evaluated with respect to several system parameters: tag number and typology, tag reciprocal distance and orientation, trajectory and speed of the moving reader antenna. The method works with a single reader antenna and without the need for any reference tag and for any arbitrary 2-D antenna path similar to those here considered. Specifically, the experimental results have shown that a mean location accuracy of the order of $10-15 \mathrm{~cm}$ can be achieved in a multi-tag scenario, when the distance of the reader from the tag and the effective synthetic aperture size are both around $1-1.5 \mathrm{~m}$, and the scan speed is less than $15 \mathrm{~cm} / \mathrm{s}$. The deviations from above-mentioned location performance, as determined by scenario geometrical parameters different from those allowed by the antenna handling system we used, can be assessed by the numerical analysis in [5]. Finally, future work will include an extensive measurement campaign to assess the performance of the SAR-based localization techniques when a robot, a drone [11] or a forklift moves the reader antenna, in more realistic warehouse scenarios. In this context, the SAR-based analysis can also be used to discriminate between moving and static tagged objects [12].

\section{REFERENCES}

[1] P. V. Nikitin, R. Martinez, S. Ramamurthy, H. Leland, G. Spiess and K. V. S. Rao, "Phase based spatial identification of UHF RFID tags," 2010 IEEE Int. Conf. RFID, Orlando, FL, 2010, pp. 102-109.

[2] M. Scherhäufl, M. Pichler, E. Schimbäck, D. J. Müller, A. Ziroff and A. Stelzer, 'Indoor Localization of Passive UHF RFID Tags Based on Phase-ofArrival Evaluation," IEEE Trans. Microwave Theory Techniques, vol. 61, no. 12, pp. 4724-4729, Dec. 2013.

[3] T. Liu, Y. Liu, L. Yang, Y. Guo and C. Wang, "BackPos: High Accuracy Backscatter Positioning System," IEEE Trans. Mobile Computing, vol. 15, no. 3, pp. 586-598, Mar. 2016.

[4] P. Nepa, F. Lombardini, A. Buffi, "Method for determining the location of a moving RFID tag," EP2533173 (A1), IP: University of Pisa, 2011.

[5] A. Buffi, P. Nepa, F. Lombardini, "A Phase-Based Technique for Localization of UHF-RFID Tags Moving on a Conveyor Belt: Performance Analysis and Test-Case Measurements," IEEE Sensors Journal, vol. 15, no. 1, pp. 387-396, Jan. 2015.

[6] A. Parr, R. Miesen and M. Vossiek, "Inverse SAR approach for localization of moving RFID tags," 2013 IEEE International Conference on RFID (RFID), Penang, 2013, pp. 104-109.

[7] A. Buffi and P. Nepa, "Advanced SARFID: A localization technique for UHF RFID tags," 2016 URSI Int. Symp. Electr. Theory, Espoo, Finland, 2016, pp. 322-325.

[8] A. Buffi and P. Nepa, "A 2D localization technique for UHF-RFID smart bookshelves," 2016 IEEE Int. Symp. Ant. Prop., Fajardo, Puerto Rico, 2016, pp. 1159-1160.

[9] R. Miesen, F. Kirsch and M. Vossiek, "UHF RFID Localization Based on Synthetic Apertures," IEEE Trans. Autom. Science Eng., vol. 10, no. 3, pp. 807-815, July 2013. 
[10] E. Di Giampaolo and F. Martinelli, "Mobile Robot Localization Using the Phase of Passive UHF RFID Signals," IEEE Trans. Ind. Elect., vol. 61, no. 1, pp. 365-376, Jan. 2014.

[11] A. Buffi, P. Nepa and R. Cioni, "SARFID on Drone: Drone-based UHF-RFID Tag Localization," to be presented at 2016 IEEE RFID Technology and Applications Conference (RFID-TA), Warsaw, Poland, September 20-22, 2017.

[12] A. Buffi and P. Nepa, "The SARFID technique for discriminating tagged items moving through a UHF-RFID gate," IEEE Sensors Journal, vol. 17, no. 9, pp. 2863-2870, May, 2017. 